\title{
Optical spectrophotometric monitoring of comet C/2006 W3 (Christensen) before perihelion
}

\author{
P. P. Korsun ${ }^{1}$, I. Kulyk ${ }^{1}$, O. V. Ivanova ${ }^{1,2}$, O. V. Zakhozhay ${ }^{1}$, V. L. Afanasiev ${ }^{3}$ \\ A. V. Sergeev ${ }^{4,5}$, and S. F. Velichko ${ }^{4,5}$

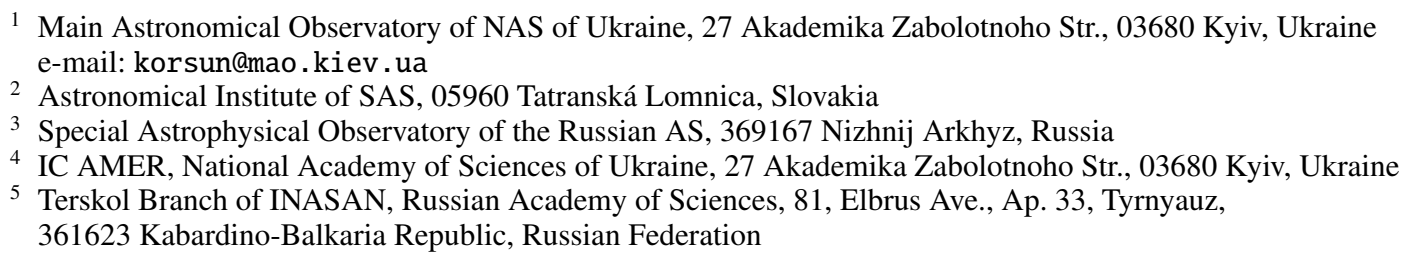

Received 2 June 2016 / Accepted 12 September 2016

\begin{abstract}
Context. Long period distant comet C/2006 W3 (Christensen) was discovered in 2006 and passed perihelion at 3.13 au in July 2009. It was a target for the Herschel Space Observatory, Spitzer Space Telescope, and ground-based radio telescopes. We enlarge the observational database with optical spectrophotometric ground base data.

Aims. We performed optical monitoring of cometary activity and searched for possible emission lines in the C/2006 W3 (Christensen) spectrum in a wide range of heliocentric distances.

Methods. We carried out pre-perihelion observations of the comet at heliocentric distances from 8.49 to 3.13 au with the $2 \mathrm{~m}$ and $6 \mathrm{~m}$ telescopes, both in imaging and spectroscopic modes, during the period 2006 to 2009 .

Results. No emissions were detected in the spectra at heliocentric distances of 8.49 and 6.25 au. Rich molecular emission spectra were obtained, when the comet was at a distance of 3.74 au from the Sun. The $\mathrm{CN}, \mathrm{C}_{3}, \mathrm{C}_{2}, \mathrm{CH}, \mathrm{NH}_{2}, \mathrm{CO}^{+}$, and, probably, $\mathrm{CH}^{+}$ emissions were assigned in the 3800-6800 $\AA$ spectral range. Gas production rates or corresponding upper limits in the emissionless case were calculated using a Haser model. We derived the gas production rates of $1.03 \times 10^{26} \mathrm{~mol} \mathrm{~s}^{-1}$ for CN, $4.12 \times 10^{24} \mathrm{~mol} \mathrm{~s}{ }^{-1}$ for $\mathrm{C}_{3}, 6.54 \times 10^{25} \mathrm{~mol} \mathrm{~s}^{-1}$ for $\mathrm{C}_{2}$, and $3.12 \times 10^{25} \mathrm{~mol} \mathrm{~s}^{-1}$ for $\mathrm{NH}_{2}$, which is matched with the $\mathrm{CN}: \mathrm{C}_{3}: \mathrm{C}_{2}: \mathrm{NH}_{2}=1.0: 0.04: 0.63: 0.30 \mathrm{gas}$ production ratios at a heliocentric distance of 3.74 au. The $\mathrm{CO}^{+}$production rate of the comet, which was equal to $2 \times 10^{24}$ mol s${ }^{-1}$, was also obtained. We quantified the evolution of the comet activity in a wide range of heliocentric distances with the $A f \rho$ technique. Heliocentric dependence of the Af $\rho$ parameters indicates that steep growth of the comet activity occurred around 3.96 au, almost simultaneously with the appearance of molecular emissions in the comet spectrum. The dust production rate was estimated from the Af $\rho$ reaching a maximal value of $380 \mathrm{~kg} \mathrm{~s}^{-1}$ at a distance of 3.13 au before perihelion passage.
\end{abstract}

Key words. comets: general - comets: individual: C/2006 W3 (Christensen)

\section{Introduction}

Comet C/2006 W3 (Christensen; hereafter W3) is a dynamically new comet. It was discovered on November 18, 2006 in Catalina Sky Survey images that were obtained with the 0.68-m Schmidt telescope at a heliocentric distance of 8.6 au (Buzzi et al. 2006). The images showed a $15^{\prime \prime}$ coma that extended slightly toward a position angle of $70-100^{\circ}$. The derived orbital parameters indicate that the comet belongs to the dynamical group of long period comets. The orbital period of W3 is about $2410000 \mathrm{yr}$, the orbital inclination to the ecliptic plane is $127^{\circ}$, indicating retrograde motion of the comet ${ }^{1}$. The comet passed perihelion on July 6, 2009 at a distance of 3.126 au. The comet W3 was already active at large distances from the Sun, thus attracting the attention of researchers. The comet brightness was about 18th magnitude when it was discovered, reaching approximately 8.5th magnitude, which is close to the perihelion. Its activity was monitored by ground-based and space telescopes from 3.66 au before perihelion until 5 au after perihelion.

\footnotetext{
1 http://ssd.jpl.nasa.gov/sbdb.cgi?sstr=2006\%20W3;old=
} $0 ; \backslash$ orb $=0 ; \operatorname{cov}=0 ; \log =0 ; \operatorname{cad}=\mathbb{0} \#$ elemq)
Atomic oxygen is an effective tracer for $\mathrm{H}_{2} \mathrm{O}, \mathrm{CO}_{2}$, and $\mathrm{CO}$ in cometary comae. Forbidden oxygen lines were measured with the ARC echelle spectrograph (ARCES) attached to the $3.5-\mathrm{m}$ Telescope at Apache Point Observatory, when the comet was close to the perihelion at 3.13 au from the Sun (McKay et al. 2012). The ratio of the green (5577 $\AA$ ) line flux to that of the red doublet (6300 and $6364 \AA$ ) was found to be $0.24 \pm 0.08$, which is considerably higher than the previous measurements for other comets at smaller heliocentric distances. This means that $\mathrm{CO}$ and/or $\mathrm{CO}_{2}$ made detectable contributions to the [OI] population in the coma of $\mathrm{W} 3$.

Spectroscopic observations, which were made at 3.66 and 3.13 au before perihelion in a wavelength range from 2.5 to $5 \mu \mathrm{m}$ provided $\mathrm{CO}: \mathrm{CO}_{2}: \mathrm{H}_{2} \mathrm{O}$ gas production ratios equal to 1.0:0.3:0.3 and 1.0:0.4:1.0 at heliocentric distances of 3.66 and $3.13 \mathrm{au}$, respectively (Ootsubo et al. 2012).

The $\mathrm{CO} / \mathrm{CO}_{2}$ ratio falls in a range of $2.5-3.5$, which considerably exceeds that obtained for other comets. The higher ratios for W3 are probably caused by insufficient sublimation of $\mathrm{CO}_{2}$ at larger distances ( $>3 \mathrm{au}$ ) from the Sun. The $\mathrm{H}_{2} \mathrm{O}$ production rate becomes significant at closer heliocentric distances. 
Reach et al. (2013) observed W3 as one of the 23 targets of the survey using the Infrared Array Camera on the Spitzer Space Telescope. The comet was observed twice: at 3.14 au before perihelion and at 4.45 au after perihelion. The broadband filters centered at 3.6 and $4.5 \mu \mathrm{m}$ enabled the distinction between dust and $\mathrm{CO}_{2}+\mathrm{CO}$ gas to be made. The authors attributed the $4.5 \mu \mathrm{m}$ flux excess to $\mathrm{CO}_{2}$ as a dominant source. However in the case of W3, $\mathrm{CO}$ is the most abundant molecule, and the comet is probably $\mathrm{CO}_{2}+\mathrm{CO}$-rich. Using this set of the data, a rotation period of $21 \mathrm{~h}$ was measured for $\mathrm{W} 3$.

The Herschel Space Observatory observed W3 at heliocentric distances from 3.3 to 5.0 au post-perihelion (Bockelée-Morvan et al. 2010; de Val-Borro et al. 2014). No lines were detected above the measured emission continuum from 70 to $672 \mu \mathrm{m}$, and the spectral energy distribution indicates thermal emission from large particles at a heliocentric distance of $3.3 \mathrm{au}$. The $\mathrm{H}_{2} \mathrm{O}$ line was observed with the Heterodyne Instrument for the Far Infrared (HIFI), when the comet was at a heliocentric distance of 5.0 au. A water production rate of $(2.0 \pm 0.5) \times 10^{27} \mathrm{~mol} \mathrm{~s}^{-1}$ was derived using a spherically symmetric radiative transfer model. The $\mathrm{NH}_{3}$ emission was not detected with an upper limit for the production rate of ammonia at $<1.5 \times 10^{27} \mathrm{~mol} \mathrm{~s}^{-1}$.

$\mathrm{CO}, \mathrm{CH}_{3} \mathrm{OH}, \mathrm{HCN}, \mathrm{H}_{2} \mathrm{~S}, \mathrm{CS}$, and $\mathrm{OH}$ spectral features were measured with the $30 \mathrm{~m}$ telescope operated by the Institute for Radio Astronomy in the Millimeter Range (IRAM) and Nançay decametric radio telescope at heliocentric distances of 3.2-3.3 au (Bockelée-Morvan et al. 2010). Water production rate was derived to be equal to $(4.2 \pm 1.0) \times 10^{28} \mathrm{~mol} \mathrm{~s}^{-1}$, assuming that water was the main source of the $\mathrm{OH}$ radicals. When normalized to $\mathrm{HCN}$, abundance ratios of the species observed with the IRAM telescope was HCN:CO: $\mathrm{CH}_{3} \mathrm{OH}: \mathrm{H}_{2} \mathrm{~S}: \mathrm{CS}=1: 240: 9: 6: 0.3$ indicating that $\mathrm{W} 3$ is $\mathrm{CO}$-rich.

Kossacki \& Szutowicz (2015), using available observations, carried out theoretical modeling and found that the nucleus of the comet should be very porous, composed of moderately fine grains that were about $10 \mu \mathrm{m}$ in diameter with a tensile strength of about $10 \mathrm{kPa}$, as was estimated for comet 9P/Tempel 1.

In our paper, we present new spectrophotometric preperihelion observations of comet W3 performed in the optical domain in a wide range of heliocentric distances, from 8.49 au (shortly after discovery) to $3.13 \mathrm{au}$. Thus, our pre-perihelion data complements and enlarges earlier findings on the comet. As a result, the comet has been observed from 8.5 au pre-perihelion to 5.0 au post-perihelion.

This paper is organized as follows. The observations are described in Sect. 2. Sections 3 and 4 give reduction techniques and results of analysis of the obtained spectroscopic and photometric data. Discussion on the derived results can be found in Sect. 4. Brief conclusions are presented in Sect. 5 .

\section{Observations}

\subsection{Photometry}

December, 2006. Spectrophotometric observations were carried out on December 12, 2006 with the multi-mode focal reducer SCORPIO attached to the prime focus of the $6 \mathrm{~m}$ Big Telescope Alt-azimuth (BTA) operated by Special Astrophysical Observatory (SAO) of the Russian Academy of Sciences (Russia). The focal reducer provided both photometric mode and long-slit spectroscopic mode (Afanasiev \& Moiseev 2005). A CCD chip EEV-42-40 of $2048 \times 2048$ pixels was used as a detector. The focal reducer converts the focal ratio to $f / 2.6$, providing a pixel scale of 0 !'18 per pixel and field of view of $6 ! 1 \times 6$. 1 . To increase the signal-to-noise ratio $(\mathrm{S} / \mathrm{N})$ of measured signal, on-chip binning of 2 in the spatial direction was applied. $1 \times 240 \mathrm{~s}$ image of the comet was obtained through a broadband $B$ filter (center wavelength $\lambda_{\mathrm{c}}=440 \mathrm{~nm}$, full width at half maximum (FWHM) $\Delta \lambda=97 \mathrm{~nm}) ; 2 \times 120 \mathrm{~s}$ images were taken through a broadband Rc filter $\left(\lambda_{\mathrm{c}}=662 \mathrm{~nm}, F W H M=150 \mathrm{~nm}\right)$. A field of standard stars (PG 0231) was observed at approximately the same airmasses as the comet to perform the photometric calibration (Landolt 1992). Reference zero points and color terms for transformation to the standard photometric system were calculated using the standard stars with a scatter of less than 0.03 mag on average. The difference between the comet and standard field airmasses was considered to be small and was compensated for with the mean monochromatic extinction coefficients measured for Peak Terskol Observatory, which is located in the same mountain region (Kulyk et al. 2004). The twilight sky was observed through the filters used to construct flat field images.

November, 2007. Another data set was obtained on November 9, 2007 with the $6 \mathrm{~m}$ Telescope. The observation set-up was similar to that described in the previous paragraph. $10 \times 10 \mathrm{~s}$ images were acquired with a broadband $V$ filter $\left(\lambda_{\mathrm{c}}=547 \mathrm{~nm}\right.$, $F W H M=79 \mathrm{~nm})$. The spectrophotometric standard star Feige 34 was observed at the similar airmasses as the comet airmasses to carry out absolute photometric calibration (Oke 1990). We used the mean monochromatic coefficient to compensate the first order of extinction, neglecting the color member, which is small in the $V$-band and comparable to the uncertainty of the main extinction itself. A set of exposures from the evening and morning twilight sky was obtained to create a flat field image.

October, 2008. To collect images of the comet, we used the 2 m RCC Telescope at Peak Terskol Observatory operated by the International Center for Astronomical, Medical and Ecological Research (IC AMER, Terskol, Kabardino-Balkaria, Russia). A focal reducer mounted at the prime focus converted the initial focal ratio of $f / 8$ to $f / 2$, providing an image scale of $0 \prime \prime 99$ per pixel and useful field of view of $9.2 \times 8.4$. The images were obtained in the $B, V, R$ color bands. However, only $R$ images were subsequently processed owing to the possible presence of an emission component in the cometary spectrum in the blue and visual regions, which was confirmed during the next observing set in December, 2008 (see below). $20 \times 30 \mathrm{~s} R$ images $\left(\lambda_{\mathrm{c}}=680 \mathrm{~nm}, F W H M=111 \mathrm{~nm}\right)$ were acquired on October 23 , 2008 when the comet was at a distance of 3.96 au from the Sun. The telescope was tracking at the comet rate, but short exposure times prevented the images of field stars from stretching. During the observation period the spectrophotometric standard stars HD 15318, HD 30739, HD 224926, and HD 188350 were observed in a wide airmass range (Bessell 1999). The standards were processed together to calculate a zero point for the instrumental magnitudes and mean coefficients of atmospheric extinction. A root mean square scatter around the regression line was $0.025 \mathrm{mag}$. The flat field images were created from the evening and morning twilling sky.

December, 2008. We observed the comet with cometary and medium band filters available in the SCORPIO device, namely, $\mathrm{CO}^{+}$and SED537. The $\mathrm{CO}^{+}$filter $\left(\lambda_{\mathrm{c}}=426.6 \mathrm{~nm}\right.$, $F W H M=6.4 \mathrm{~nm}$ ) isolates $\mathrm{CO}^{+}$emission and the underlying continuum, while the SED537 filter $\left(\lambda_{\mathrm{c}}=530.9 \mathrm{~nm}\right.$, $F W H M=16.9 \mathrm{~nm}$ ) isolates the continuum. The telescope tracked the comet to compensate its apparent movement during the observations. We observed the spectrophotometric standard star BD+25d4655 to fulfill absolute calibration (Oke 1990). Spectral atmospheric transparency at the Special Astronomical 
Table 1. Journal of C/2006 W3 observations.

\begin{tabular}{|c|c|c|c|c|c|c|c|c|}
\hline $\begin{array}{l}\text { Start of exposition } \\
\text { (UT) }\end{array}$ & $\begin{array}{c}\text { Exposition } \\
\text { (s) }\end{array}$ & $\begin{array}{c}\Delta \\
(\mathrm{au})\end{array}$ & $\begin{array}{c}r \\
(\mathrm{au})\end{array}$ & $\begin{array}{c}\text { Phase angle } \\
\left({ }^{\circ}\right)\end{array}$ & $\begin{array}{c}\text { Air } \\
\text { mass }\end{array}$ & $\begin{array}{l}\text { Data } \\
\text { type }\end{array}$ & $\begin{array}{c}\text { Filter / } \\
\text { Wavelength range }(\AA)\end{array}$ & Telescope \\
\hline $2006 / 12 / 13.8104$ & $2 \times 120$ & 7.62 & 8.49 & 3.26 & 1.17 & image & $R \mathrm{c}$ & 6-m (SAO) \\
\hline 2006/12/13.8141 & 240 & 7.62 & 8.49 & 3.26 & 1.16 & image & $B$ & 6-m (SAO) \\
\hline $2006 / 12 / 13.8186$ & 900 & 7.62 & 8.49 & 3.25 & 1.16 & spectrum & $3800-7200$ & 6-m (SAO) \\
\hline 2007/11/09.9429 & $8 \times 30$ & 5.63 & 6.24 & 7.51 & 1.13 & image & $V$ & 6-m (SAO) \\
\hline $2007 / 11 / 09.9510$ & $3 \times 900$ & 5.63 & 6.25 & 7.52 & 1.17 & spectrum & $3800-7200$ & 6-m (SAO) \\
\hline $2008 / 10 / 23.6850$ & $11 \times 30$ & 3.45 & 3.96 & 13.22 & 1.18 & image & $R$ & 2-m (Terskol) \\
\hline $2008 / 12 / 03.8326$ & $3 \times 600$ & 3.41 & 3.74 & 14.92 & 1.41 & spectrum & $3800-5400$ & 6-m (SAO) \\
\hline $2008 / 12 / 03.9361$ & $11 \times 60$ & 3.41 & 3.74 & 14.92 & 1.51 & image & $\mathrm{CO}^{+}$ & 6-m (SAO) \\
\hline 2008/12/03.9529 & $10 \times 30$ & 3.41 & 3.74 & 14.92 & 1.58 & image & SED537 & 6-m (SAO) \\
\hline $2008 / 12 / 04.7754$ & $3 \times 600$ & 3.41 & 3.74 & 14.97 & 1.21 & spectrum & $3800-7200$ & 6-m (SAO) \\
\hline $2009 / 06 / 22.9346$ & $21 \times 180$ & 2.85 & 3.13 & 18.84 & 1.22 & image & bc & 2-m (Terskol) \\
\hline $2009 / 06 / 22.9773$ & $19 \times 180$ & 2.85 & 3.13 & 18.84 & 1.09 & image & $\mathrm{rc}$ & 2-m (Terskol) \\
\hline
\end{tabular}

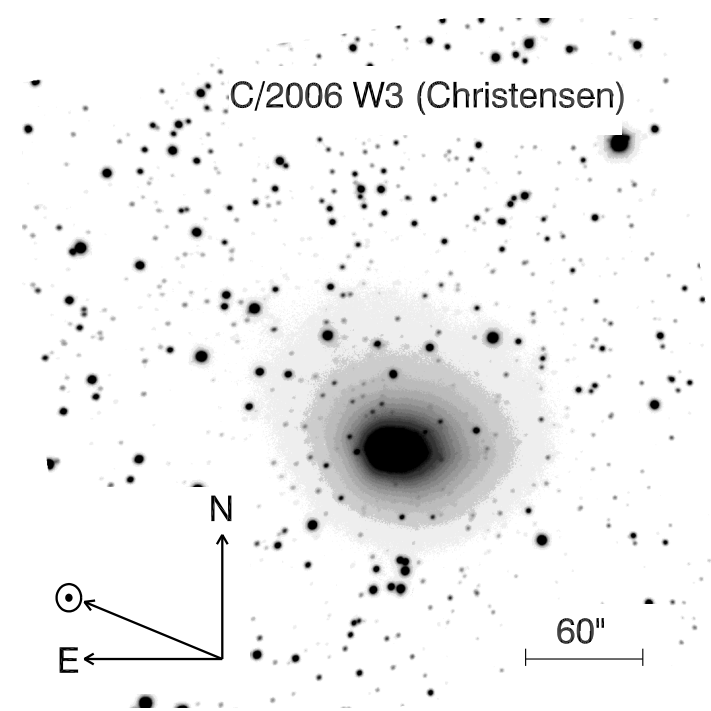

Fig. 1. Image of $\mathrm{W} 3$ obtained through $R$-filter with the $6 \mathrm{~m}$ telescope on December 4, 2008. North $(\mathrm{N})$, East $(\mathrm{E})$, sunward direction $(\odot)$, and scale bar are indicated.

Observatory was calculated from the measurements presented by Kartasheva \& Chunakova (1978). The morning sky provided the set of flat field images for correction of the scientific frames. Figure 1 shows the appearance of the comet in December 2008, when it was at a heliocentric distance of $3.74 \mathrm{au}$.

June, 2009. W3 was observed with the $2 \mathrm{~m}$ RCC Telescope at Peak Terskol on June 22, 2009. The focal reducer mounted at the Cassegrain focus converted the focal ratio to $f / 2.2048 \times 2048$ CCD detector with a pixel size of $24 \mu \mathrm{m}$, which provided an image scale $0^{\prime} .31$ per pixel and field of view of about $10,8 \times 10 \prime 8$. The telescope was tracked at the nonsidereal motion to compensate for the apparent movement of the comet. Two narrowband filters, bc and rc, designed by ESO were used to measure cometary dust: a blue continuum at $443.0 \mathrm{~nm}(F W H M=$ $4.0 \mathrm{~nm})$ and red continuum at $684.0 \mathrm{~nm}(F W H M=9.0 \mathrm{~nm})$ (Schleicher \& Farnham 2004). $40 \times 180$ s exposures were obtained in the $B$ and $R$ bands. The spectrophotometric standard stars HD 188350 and HD 129956 were observed at different airmasses to derive monochromatic extinction coefficients and instrumental zero points. A scatter from the least square fit to the standard star measurements was equal to 0.03 mag on average. The set of flat field images were obtained during twilights.

\subsection{Spectroscopic observations}

Two-dimensional spectra of the comet were obtained with the focal reducer SCORPIO, mounted at the prime focus of the $6 \mathrm{~m}$ telescope BTA (SAO RAS). SCORPIO was equipped with an EEV-42-40 CCD detector of $2048 \times 2048$ pixels, providing a field of view of $6.1 \times 6.1$ with a scale of 0".18/pixel (Afanasiev \& Moiseev 2005). The VPHG1200B and VPHG550G transparent grisms were used as dispersers. The spectral resolution, which was defind by a slit width of 0.5 , was $2.5 \AA$ and $5 \AA$ using the VPHG1200B and VPHG550G grisms, respectively.

To fulfill absolute calibration the spectrophotometric standard stars G191b2b, Feige 34, and BD+25d4655 were exposed (Oke 1990). Variations in the wavelength dependence of atmospheric extinction were taken from Kartasheva \& Chunakova (1978). During exposures, the telescope tracked the apparent speed of the comet, compensating for its displacement relative to the field stars. A log of the observations is presented in Table 1.

\section{Photometry}

\section{1. $\mathrm{CO}^{+}$morphology of the comet}

The $\mathrm{CO}^{+}$emissions were detected from our spectroscopic observations at a heliocentric distance of $3.74 \mathrm{au}$. Therefore, there was a motivation to examine an extension of $\mathrm{CO}^{+}$within the coma. The $\mathrm{CO}^{+}$in a comet can be produced by photoionization of $\mathrm{CO}$, by dissociative ionization of $\mathrm{CO}_{2}$, and by an extended source, possibly related to the dust. Also $\mathrm{CO}^{+}$can arise from the charge exchange with solar wind protons (Krishna Swamy 2010).

The ion coma of the comet was obtained by substraction of the coadded continuum image obtained through the SED537 filter from the coadded $\mathrm{CO}^{+}$image. For correct subtracting, we took into account the different light transmission of the filters and the different continua levels. We also took into account the available reddening, which was taken from our spectroscopic measurements of $\mathrm{W} 3$. The isolated $\mathrm{CO}^{+}$coma of $\mathrm{W} 3$ is presented in Fig. 2a. It is slightly elongated in the direction opposite to the Sun.

The ion image was transformed into the column densities using the $g$-factor provided by Lutz et al. (1993). In Fig. 2, the contour line equal to $1.99 \times 10^{-17} \mathrm{erg} \mathrm{cm}^{-2} \mathrm{~s}^{-1} \AA^{-1}$ corresponds to a column density of $3 \times 10^{7}$ particles per $\mathrm{cm}^{2}$ at a cometocentric distance of $25000 \mathrm{~km}$. The column density equal to $6 \times 10^{10}$ particles per $\mathrm{cm}^{2}$ at a distance of approximately $100000 \mathrm{~km}$ from 

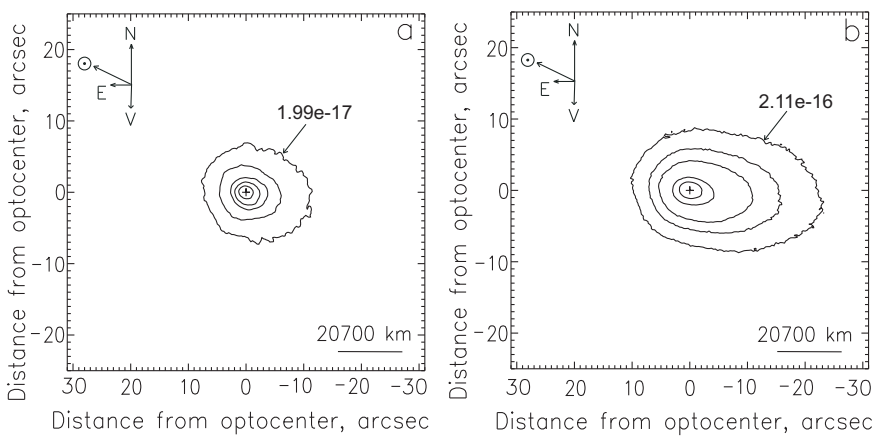

Fig. 2. $\mathrm{CO}^{+}$a) and dust b) comae of $\mathrm{W} 3$ (December 3.9, 2008). The outmost contours are indicated. Both images are scaled in erg cm $\mathrm{cm}^{-2} \mathrm{~s}^{-1} \AA^{-1}$ North $(\mathrm{N})$, East $(\mathrm{E})$, sunward $(\odot)$, and motion $(V)$ directions are denoted.

the comet nucleus was derived for comet 29P/SchwassmannWachmann 1, which was at a distance of 5.77 au from the Sun (Jockers et al. 1992); and $1.3 \times 10^{7}$ particles per $\mathrm{cm}^{2}$ at a cometocentric distance of $72000 \mathrm{~km}$ were obtained for comet C/2002 VQ94 (LINEAR), which was observed at a heliocentric distance of 7.34 au (Korsun et al. 2008).

A crude estimate of the $\mathrm{CO}^{+}$production of the comet can be obtained from the product of the integral of the column density across the comet tail projection (i.e. the tail ion content per unit tail length) and the ion flow velocity projected into the sky plane (Jockers et al. 1992). Using an approach described by Jockers et al. (1992), we estimated the $\mathrm{CO}^{+}$production rate using values of a mean column density across the tail projection [particles $\mathrm{cm}^{-2}$ ], tail width [cm], and ion velocity [ $\mathrm{cm} \mathrm{s}^{-1}$ ], projected into the sky plane. The mean column density for the comet was $2 \times 10^{8}$ particles per $\mathrm{cm}^{2}$ and the tail width was $4 \times 10^{9} \mathrm{~cm}$. We also estimated the velocity projected into the sky plane for the comet to $30 \mathrm{~km} \mathrm{~s}^{-1}$. We assumed that the ion velocity was about $100 \mathrm{~km} \mathrm{~s}^{-1}$, the phase angle of the comet at the moment of observation was $14 \% 9$, and the angle between the tail axis and the line of sight was $5^{\circ}$ (corresponding to average aberration angles of plasma tails, Krishna Swamy 2010). We obtained $2 \times 10^{24} \mathrm{CO}^{+}$particles per second.

\subsection{Dust continuum}

\subsubsection{Image analysis}

The images were pre-processed by subtracting a bias (zero exposure) image and then divided by a flat field image. The latter was created as a median of the appropriative exposures taken during twilights. We used IDL library routine sky.pro to calculate the local background intensity (Stetson 1987). Aperture photometry was performed on all standard stars using circular apertures with large radii that were approximately equal to $3 \times F W H M$. Photometric uncertainty, $\sigma$, was computed as $\sigma=\left(\sigma_{\text {stat }}^{2}+\sigma_{\mathrm{k}}^{2}\right) / 2$, where $\sigma_{\text {stat }}$ and $\sigma_{\mathrm{k}}$ are respectively the statistical error that is dominated by background uncertainty, and the scatter in the photometric transformation coefficients deduced from the standard star images. To evaluate $\sigma_{\text {stat }}$ the $\mathrm{S} / \mathrm{N}$ equation was taken from Merline \& Howell (1995). The noise model takes into account the number of pixels in the apertures used for the target and background integration as well as readout noise. Typically, $\sigma_{\text {stat }}$ varied with the brightness of the object and did not exceed $1 \%$, and $\sigma_{\mathrm{k}}$ was estimated to be between 1 and $3 \%$ (see previous section). Since the mean monochromatic extinction coefficients were used (see Sect. 2.1) a systematic error could be introduced into the calibrated magnitudes. We minimized this effect during the observations, choosing the standards as close as possible to the target. Considering an upper limit of $0.1 \mathrm{mag} /$ airmass on uncertainty of the mean extinction coefficient and 0.3 airmass difference, the possible systematic effect on the derived magnitudes did not exceed 0.03 mag. The surface brightness profiles of the target were analyzed by comparing them with the profile of a star. For this reason, average images were constructed for each night: one for the target and one for a reference star chosen to have a good $\mathrm{S} / \mathrm{N}$ and no other star too close to it. In both cases, we shifted the individual images in such a way as to put the target/star in the center, and then the individual images in each group were stacked together. The median filtration was applied to remove faint background stars and possible artifacts. Close bright stars were masked with the neighbor background signal.

Photometric profiles were created by calculating brightness averages within concentric circular annuli centered at the photometric centers of the target and the star. The comet's total brightness was measured within a series of apertures with different radii. The photometric results in this paper are based on the reference aperture encapsulating the entire coma. An aperture radius of $10^{\prime \prime}$ projected at the distant of the comet corresponded roughly to $55000-21000 \mathrm{~km}$, depending on the period of observations.

The examined surface brightness profiles of the comet clearly indicate the evolution of the coma during the preperihelion monitoring between heliocentric distances, 8.49 and $3.13 \mathrm{au}$. The featureless spectra of the comet obtained in 2006 and 2007 enabled us to use broadband filters to estimate the dust production rate of the nucleus and an upper limit on the nucleus size. In 2008 emission features had not yet been recorded in the red region of a visual spectrum, therefore the $R$-filter images were used to quantify the dust production rate. In 2009, the dust environment of W3 was investigated by isolating the blue and red continuum regions with corresponding filters.

An $A f \rho$ technique was used as a proxy for the dust production rate (Ahearn et al. 1984). Af $\rho$ is a product of the Bond albedo $(A)$, filling factor $(f)$ within the aperture field of view (that is the number of particles times their mean cross-section and divided by the area of the field of view), and the linear radius of the aperture, $\rho$, projected on the sky-plane at the object distance

$A f \rho=4 \frac{\Delta^{2} r^{2}}{\rho} \frac{F_{\text {obj }}}{F_{\text {sun }}}$.

Here, $\Delta$ and $r$ are the geocentric and heliocentric distances, respectively; $F_{\text {sun }}$ is the solar flux at 1 au convolved with the filtertransmitting curve; $F_{\text {obj }}$ is the object continuum flux measured within the given aperture. $\Delta$ and $\rho$ must be expressed in the same unit $(\mathrm{cm})$ and $\mathrm{r}$ in au. A simple steady-state dust outflow model implies the constant dust production rate from a nucleus and the constant velocity of ejected particles, therefore the product of $A f$ and $\rho$ should be aperture independent and can be used to estimate dust production.

Considering that the brightness of an active object is the sum of the scattered light from the coma and nucleus, care must be taken in using $A f \rho$ if activity level is low. The shape of the surface brightness distribution at the telescope focal plane depends on relative contribution of the coma and nucleus fluxes into the total intensity (Tancredi et al. 2000). Assuming a nucleus size of $10 \mathrm{~km}$ and albedo 0.04, the flux from the nucleus should contribute about $9 \%$ to the total flux of the comet in 2006 and be negligible for other observing periods (Bockelée-Morvan et al. 2010). The brightness surface profiles of W3 and a field star 
Table 2. Photometry results: the total magnitudes, $A f \rho$, logarithmic surface brightness gradients.

\begin{tabular}{lccccc}
\hline \hline Date & $r$, au & Filter & $M^{a}, \mathrm{mag}$ & $A f \rho, \mathrm{cm}$ & Slope \\
\hline $2006 / 12 / 13.8090$ & 8.49 & $R c$ & $18.41 \pm 0.01$ & $400 \pm 20$ & $-1.55 \pm 0.01$ \\
$2007 / 11 / 09.9429$ & 6.24 & $V$ & $15.64 \pm 0.01$ & $2000 \pm 50$ & $-1.46 \pm 0.01$ \\
$2008 / 10 / 23.6850$ & 3.96 & $R$ & $12.36 \pm 0.01$ & $5900 \pm 300$ & $-1.24 \pm 0.01$ \\
$2009 / 06 / 22.9346$ & 3.13 & bc & $12.19 \pm 0.01$ & $9000 \pm 400$ & $-1.19 \pm 0.01$ \\
$2009 / 06 / 22.9776$ & 3.13 & rc & $11.15 \pm 0.01$ & $11600 \pm 500$ & $-1.11 \pm 0.01$ \\
\hline
\end{tabular}

Notes. ${ }^{(a)}$ Derived from the apertures collecting flux from the coma, $\rho \sim 5 \times F W H M$.

were used to estimate the flux from the nucleus in 2006, assuming that it is represented with a Gaussian-like PSF (Point Spread Function) similar to star images from the same field (Kulyk et al. 2016). The estimated flux from the nucleus allowed us to put an upper limit on the nucleus size using the following expression (Russell 1916):

$A R_{\mathrm{N}}^{2}=\frac{10^{22} r^{2} \Delta^{2} 10^{\left(0.4\left(m_{\mathrm{sun}}-m\right)\right.}}{10^{-0.4(\alpha \beta)}}$

where $A$ is the geometric albedo, $R_{\mathrm{N}}[\mathrm{m}]$ is the target radius, $r[\mathrm{au}]$ and $\Delta[\mathrm{au}]$ are the heliocentric and geocentric distances, $m_{\text {sun }}$ and $m$ are the apparent magnitudes of the Sun and target in the certain filter, $\alpha$ is the phase angle, and $\beta$ is the phase coefficient. The solar magnitude for each filter was calculated using the solar spectrum and the spectrum of a standard star taken from Oke (1990) with subsequent transformation to the standard Johnson-Morgan-Cousins system (Neckel \& Labs 1984; Fukugita et al. 1996). The geometric albedo was fixed at 0.04 . We assumed the commonly used value of $0.04 \mathrm{mag} / \mathrm{deg}$ for the phase coefficient (Lamy et al. 2004). Adopting an albedo value 0.04 and uncertainty \pm 0.2 and \pm 0.1 of the albedo and measured magnitudes, the uncertainty of the radius estimate would be $22 \%$ (Tancredi et al. 2000).

In 2009, images were obtained through the blue and red continuum windows, therefore we used them not only to derive $A f \rho$ but also to examine the spatial distribution of the dust color. The dust color was defined as the normalized reflectivity gradient following Jewitt \& Meech (1986):

$S^{\prime}\left(\lambda_{1}, \lambda_{2}\right)=\frac{20}{\Delta \lambda} \frac{10^{0.4\left(C I_{\mathrm{com}}-C I_{\mathrm{sun}}\right)}-1}{10^{0.4\left(C I_{\mathrm{com}}-C I_{\mathrm{sun}}\right)}+1}$,

where $S^{\prime}[\% / 1000 \AA]$ is the normalized reflectivity, $\Delta \lambda$ is the difference of effective wavelengths of the filters measured in microns, and CI is the target and the solar color indices measured in magnitudes. Red and blue continuum images were calibrated to absolute intensities and the color map was constructed following Bonev et al. (2002).

\subsubsection{Results}

Surface brightness profiles of $\mathrm{W} 3$ are depicted in Fig. 3. The figure clearly shows that the comet was already active from our earliest observation in December 2006 at a heliocentric distance of $8.49 \mathrm{au}$. The calculated magnitude, the logarithmic surface brightness gradient, and the $A f \rho$ parameter are summarized in Table 2 for each observing run. Following the $A f \rho$ notation, $A f \rho$ should be aperture-independent and, in this case, it can be used to estimate dust production. Under steady conditions, the volume density of emitted grains is in inverse proportion to the squared distance from a nucleus $N_{\mathrm{d}} \sim 1 / \rho^{2}$ and the observed radial surface brightness of continuum is expected to decrease as $\rho^{-1}$.
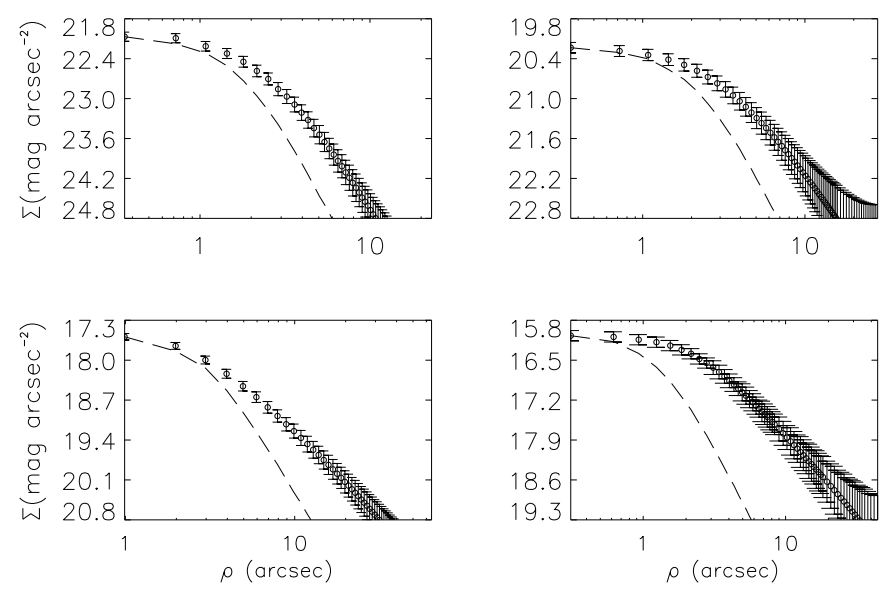

Fig. 3. Surface brightness profiles of $\mathrm{W} 3$ for the images obtained in 2006, 2007 (upper panel), 2008, and 2009 (lower panel). The points correspond to the comet profiles, the dashed lines represent the star PSF.

A log-log fit to the surface brightness points out that the profiles of W3 become shallower when the distance to the Sun decreases. The slope of the surface brightness exceeds the canonical case of a steady-state coma with a slope of -1.0 for all observing periods falling between -1.0 and -1.5 .

The total magnitudes and $A f \rho$ parameters were calculated with large apertures including the area of the coma with a relatively good S/N: approximately $10^{\prime \prime}$ and $20^{\prime \prime}$ for the data obtained in 2006-2007 and in 2008-2009, respectively. Uncertainty of the $A f \rho$ parameters, 6-10\%, was dominated by the photometric errors of the measured magnitudes.

Figure 4 shows the measured $A f \rho$ parameter as a function of the heliocentric distance. A scale of the spherical blackbody equilibrium temperature that corresponds with the heliocentric distances is also presented in the figure.

A quantitative estimate of dust production of W3's nucleus was made from the calculated $A f \rho$ parameters. We used an approach developed by Newburn \& Spinrad (1985) and Singh et al. (1992). This requires several assumptions about the physical characteristics of dust particles in the coma. To calculate the dust production rate, we considered the radii of dust grains falling into the intervals $0.1-100 \mu \mathrm{m}$ and $0.1-$ $600 \mu \mathrm{m}$ for data obtained in 2006/2007 and 2008/2009, respectively (de Val-Borro et al. 2014). The differential size distribution of grains was described by a differential power law of index -3.6 (Bockelée-Morvan et al. 2010). The size-dependent terminal grain velocity was computed following Sekanina et al. (1992). Taking into account the heliocentric distance dependence, the grain velocities $v(r)=3 \mathrm{~m} \mathrm{~s}^{-1}$ and $v(r)=250 \mathrm{~m} \mathrm{~s}^{-1}$ were derived for the limiting particle sizes $a=600 \mu \mathrm{m}$ and $a=0.1 \mu \mathrm{m}$, respectively. 


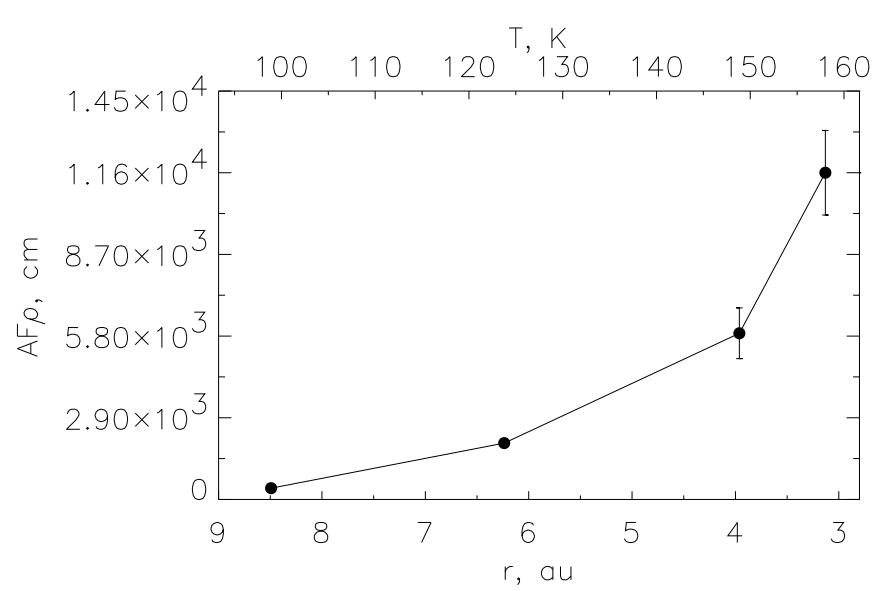

Fig. 4. Pre-perihelion level of W3 activity versus heliocentric distance. The upper horizontal axis scales the spherical blackbody equilibrium temperature, corresponding to the heliocentric distances.

Table 3. Dust production rate of W3.

\begin{tabular}{cccc}
\hline \hline$r, \mathrm{au}$ & $a_{\mathrm{max}}, \mu \mathrm{m}$ & $v(r), \mathrm{m} \mathrm{s}^{-1}$ & $Q, \mathrm{~kg} \mathrm{~s}^{-1}$ \\
\hline 8.49 & 100 & $150-5$ & 3 \\
6.24 & 100 & $170-2$ & 20 \\
3.96 & 600 & $220-3$ & 130 \\
3.13 & 600 & $250-3$ & 380 \\
\hline
\end{tabular}

The size-dependent densities were computed following an expression $\rho_{\mathrm{d}}(a)=\rho_{0}-\rho_{1}\left(a /\left(a+a_{2}\right)\right)$ with $\rho_{0}=3.0 \mathrm{~g} \mathrm{~cm}^{-3}$, $\rho_{1}=2.2 \mathrm{~g} \mathrm{~cm}^{-3}$, and $a_{2}=2 \mathrm{~mm}$. This gives a density of $1.3 \mathrm{~g} \mathrm{~cm}^{-3}$ to the smallest particles and a density of $0.8 \mathrm{~g} \mathrm{~cm}^{-3}$ to the largest particles, which are thought to be more fluffy and porous (Newburn \& Spinrad 1985). We fixed average geometric albedo in a given wavelength region at 0.05 , considering a phase function in the form of $10^{-0.4 \beta \alpha}$ with a phase coefficient fixed at 0.04 . The calculated dust production rates, $Q$, are summarized in Table 3, along with the minimal and maximal grain velocities.

An upper limit of the nucleus radius was estimated from the $\mathrm{R}$ images that were obtained in 2006. After subtracting a portion of the flux contributed by the coma, the so-called nucleus flux was integrated within circular apertures and converted into the magnitudes. We estimated an upper limit of the nucleus $R_{\mathrm{N}}=$ $13 \pm 2 \mathrm{~km}$ (see Eq. (2)), which is in agreement with the diameter $<26 \mathrm{~km}$ estimated by Bockelée-Morvan et al. (2010).

Colors of the coma were computed with Eq. (3) from the $B$ and $R$ narrow-band continuum images obtained in 2009. To create a color map, we used the area around the photometric center of the comet where the signal from the coma was more than three times higher than the standard deviation of the sky noise for both images in the BR pair. Owing to this restriction, the color map extends to a much smaller distance than the $R$ surface brightness, which is even smaller than the $B$ surface brightness. Dependence of the normalized reflectivity gradient on azimuth is depicted in Fig. 5. For each azimuth value, the graph shows four values of the normalized reflectivity gradient expressed in $\% / 1000 \AA$, which are a mean within fixed radius vector intervals. The solid vertical line indicates the antisolar direction. The normalized reflectivity gradient of the inner coma varies between $2 \% / 1000 \AA$ and $5 \% / 1000 \AA$, increasing to $10 \% / 1000 \AA$ in the outer part of the coma. The color of the inner part of the W3 coma is in accord with the sample of long period comets, particularly, with the color of C/1995 O1(Hale-Bopp) measured

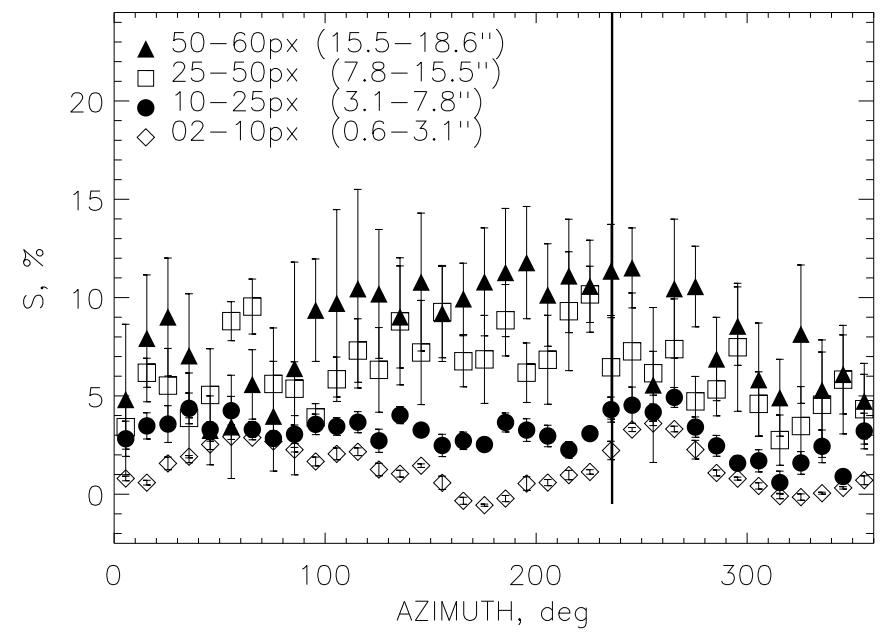

Fig. 5. Azimuthal stretch of the normalized spectral gradient of the W3 coma. Four values are depicted for each azimuth, which are averaged within the fixed intervals of radius-vectors (shown as a legend). Uncertainties are $3 \sigma$ rms errors computed within the intervals.

between 3 and 13 au from the Sun (Jewitt 2015; Weiler et al. 2003). The reddening of outer regions of the coma was found for comets C/LINEAR (2000 WM1) by Bonev \& Jockers (2002) and C/2003 WT42 (LINEAR) by Korsun et al. (2010).

\section{Spectroscopy}

\subsection{Spectra reductions}

The software codes used for processing the spectra include subtraction of the averaged frame of bias, correction for geometry of the spectra, interframe median filtering to remove traces of cosmic rays on frames, and absolute calibration of the observed spectra. Spectra of a lamp with smooth distribution of energy in the spectrum were used to provide heterogeneity of pixel sensitivity (flat fielding). The night sky spectrum was removed by polynomial fitting of the sky background in each column over the zones that were free of the target's coma. To establish the wavelength scale along dispersion exposures of an He-Ne-Ar lamp were used. Finally, two-dimensional spectra were collapsed in a spatial direction to build one-dimensional ones. The signal was integrated in a spatial direction, where the signal from the coma was above the signal $/$ noise $=1$ level.

\subsection{Energy distribution along dispersion}

Spectra of comets generally consist of two main components: continuum caused by the scattering of sunlight by dust particles and emissions due to reemitting of solar quantums by molecules in the cometary coma. To separate these components, we fitted the observed continuum using a high-resolution solar spectrum (Neckel \& Labs 1984). It was degraded to the resolution of our observations by convolving with the appropriate instrumental profile and normalized to the flux from the comet around $5000 \AA$ A. A proper wavelength dependence was gained by multiplying the solar spectrum by a polynomial. The fitted continua and the observed spectra are depicted in Fig. 6.

Visual inspection does not reveal emissions in the spectra obtained in 2006 and 2007, when the comet was on the heliocentric distances of 8.49 and 6.25 au, respectively, while the emissions clearly appeared in 2008 , when the comet was at 3.74 au 
P. P. Korsun et al.: Optical monitoring of comet C/2006 W3 (Christensen)

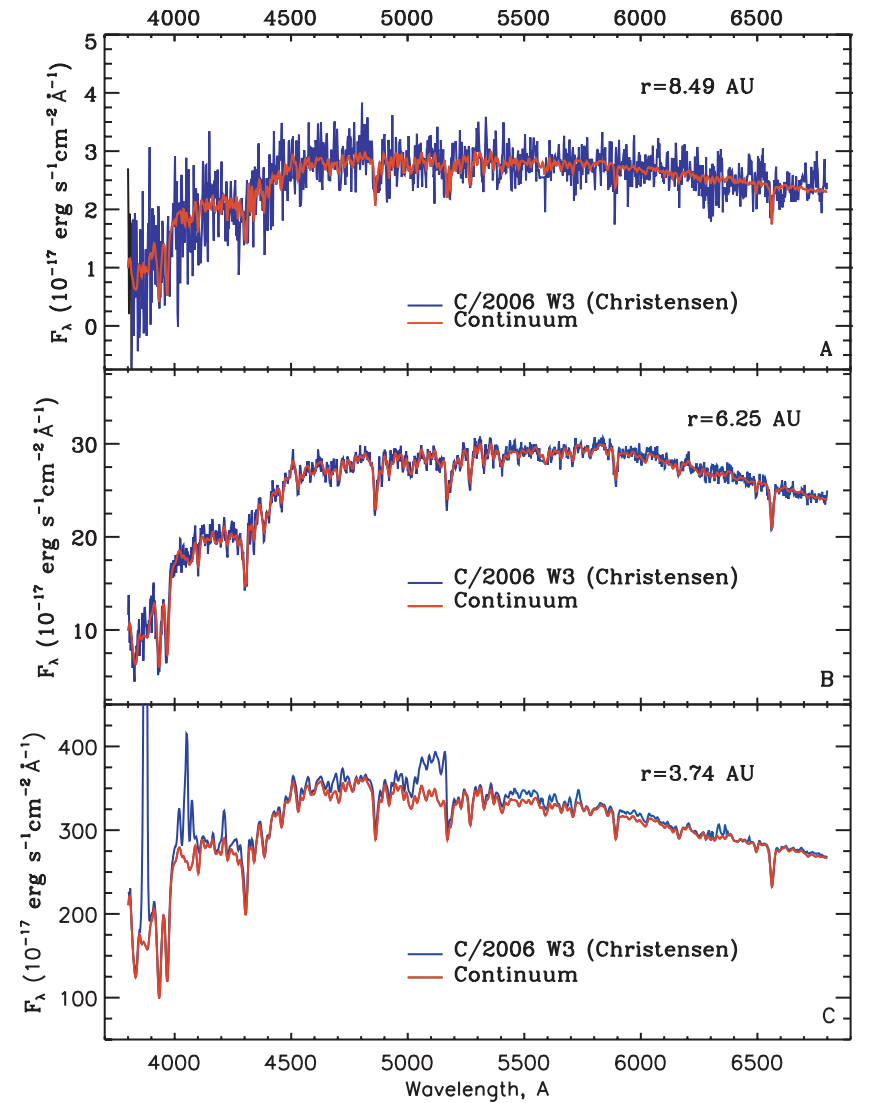

Fig. 6. Evolution of the spectra of W3, when the comet approached the Sun from 8.49 to 3.74 au and fitted continua. Molecular emissions are clearly seen in the $\mathbf{C}$ ) panel.

inbound to the Sun. The W3 spectrum with the emission component is presented in Fig. 7, where the fitted continuum is subtracted. The local $3 \sigma$-levels, where the strongest molecular emissions are expected, are also marked above the noise signal in Fig. 7A, B. The result, obtained in December 2008, unexpectedly differs from emissionless spectra: these show a rich emission component. Most known emissions in optical domain were detected in the spectra. The spectrum obtained on December 4, 2009 covers $3800-6800 \AA$ with a spectral resolution of $5 \AA$. We unambiguously detected vibration bands of the molecules: $\mathrm{CN}$ Violet system $(\Delta v=0$ and $\Delta v=-1), \mathrm{C}_{3}$ group, $\mathrm{C}_{2}$ Swan bands $(\Delta v=+2, \Delta v=+1, \Delta v=0, \Delta v=-1$, and $\Delta v=-2), \mathrm{CH}, \mathrm{NH}_{2}$, and $\mathrm{CO}^{+}$Comet-tail bands (3-0, 2-0, 4-2, 1-0, and 0-0). These are shown in Fig. 7C. The identifications are confirmed by the spectrum obtained on December 3, 2008 with a spectral resolution of $2.5 \AA$ in the wavelength range of 3800-5400 $\AA$. Mostly this related to the identification of the weak emissions such as $\mathrm{CH}, \mathrm{NH}_{2}$, and $\mathrm{CO}^{+}$, so we limited the flux axis in Fig. 7D, to verify our findings. More detailed assignments of the emission features are marked in Fig. 7D. Here, and in Fig. 7C, we also indicated a feature around $4232 \AA$ as $\mathrm{CH}^{+}$. With one emission peak, we have no strict evidence of this assignment. Nevertheless, emissions of the neutral molecule $\mathrm{CH}$ are detected in the spectra.

\subsection{Gas production rates}

A simple Haser model, which assumes isotropic outgassing at constant speed and photodissociation of the parent and daughter
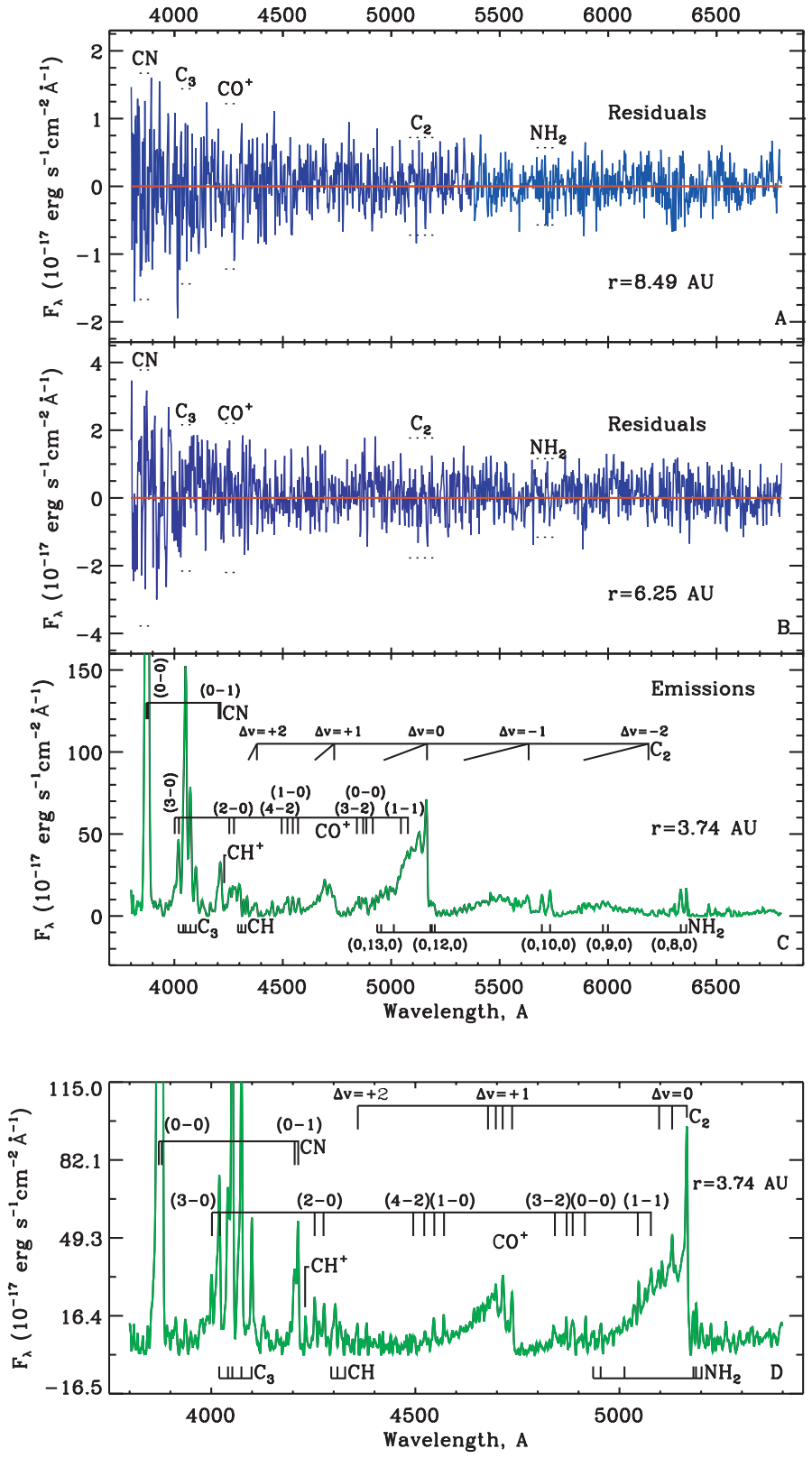

Fig. 7. The observed spectrum - fitted continuum of W3. No emissions above the $3 \sigma$-levels were detected, when the comet was at heliocentric distances greater than 6.25 au from the Sun (Panels A) and B)). Emissions are identified in the spectra, when the comet was at a heliocentric distance of 3.74 au (Panels C) and D)).

molecules was used to derive production rates of the radicals detected in the cometary spectra (Haser 1957):

$N_{\mathrm{d}}(\rho)=\frac{Q}{4 \pi v \rho} \frac{l_{\mathrm{d}}}{l_{\mathrm{p}}-l_{\mathrm{d}}}\left(\int_{0}^{\frac{\rho}{l_{\mathrm{p}}}} K_{0}(y) \mathrm{d} y-\int_{0}^{\frac{\rho}{l_{\mathrm{d}}}} K_{0}(y) \mathrm{d} y\right)$.

Here, $\rho$ is the projected distance from the nucleus through which the line-of-sight integration is performed, $l_{\mathrm{p}}$ and $l_{\mathrm{d}}$ are the parent and daughter scale lengths given by the product of the radial outflow speed, $v$, and exponential lifetimes of the parent of the observed species, $N_{\mathrm{d}}(\rho)$ and $Q$ are the density and production rate of a daughter species, and $K_{0}(y)$ is the modified Bessel function of the first kind. 
Table 4. Parameters used to determine the gas production rates (for $r=1 \mathrm{au})$.

\begin{tabular}{lccc}
\hline \hline Molecule & $\begin{array}{c}g \text {-factor } \times 10^{-13} \\
\left(\mathrm{erg} \mathrm{s}^{-1} \mathrm{~mol}^{-1}\right)\end{array}$ & $\begin{array}{c}t_{\mathrm{p}} \\
\left(10^{4} \mathrm{~km}\right)\end{array}$ & $\begin{array}{c}t_{\mathrm{d}} \\
\left(10^{4} \mathrm{~km}\right)\end{array}$ \\
\hline $\mathrm{CN}(0-0)$ & $g_{\mathrm{CN}}{ }^{a}$ & 1.7 & 30. \\
$\mathrm{C}_{3}$ & 10.0 & 0.31 & 15. \\
$\mathrm{C}_{2}(\Delta v=0)$ & 4.47 & 2.5 & 12. \\
$\mathrm{NH}_{2}$ & 0.19 & 0.41 & 6.2 \\
\hline
\end{tabular}

Notes. ${ }^{(a)} g_{\mathrm{CN}}$ is equal to 3.35 for data obtained in 2006-2007 and 3.65 for data obtained in 2008.

The predominant mechanism of molecular emissions in the cometary comae is the resonance fluorescence. To convert the observed fluxes $F(\rho)$ to the column density, we can use the known values of the fluorescence efficiency ( $g$-factors) for the corresponding molecular bands. In this case, the following relation is true:

$N_{\mathrm{d}}(\rho)=\frac{4 \pi}{g} \frac{F(\rho)}{\Omega}$.

The $g / 4 \pi$ factor corresponds to the amount of energy that is reemitted by one molecule per second to a solid angle equal to one steradian. $\Omega$ is the solid angle, which is determined by the projection of a unit element of the used CCD on the celestial sphere.

Gas production rates derived with a Haser model are modeldependent. To calculate production rates, we adopted model parameters that were used for interpretation of the spectra of comet C/1995 O1 (Hale-Bopp) when it was at large heliocentric distances before perihelion (Rauer et al. 1997). Original data were taken from publications referring in this article (A'Hearn et al. 1995; Cochran et al. 1992). The $g$-factor for $\mathrm{CN}, g_{\mathrm{CN}}$, which varies with the heliocentric velocity of the comet, was taken from Schleicher (2010). A heliocentric dependence of the parent and daughter scale lengths was scaled by $r^{-2}$ and that for $g$-factors by $r^{2}$. We used a gas outflow velocity of $1 \mathrm{~km} \mathrm{~s}^{-1}$ at 1 au and scaled it to the observed distance by $r^{-0.5}$.

We did not detect any emissions in the spectra that was obtained in 2006 and 2007. Therefore, we determined only upper limits to the fluxes of $\mathrm{CN}, \mathrm{C}_{3}, \mathrm{C}_{2}$, and $\mathrm{CO}^{+}$and upper limits to the production rate of the neutrals. To obtain the upper limits to the emission fluxes, we used an approach that was applied to the emission-free spectra of distant comet C/2006 S3 (LONEOS) (Rousselot et al. 2014). The amplitudes of the minimal measurable signal, which are equal to the rms noise level, were calculated within wavelength regions associated with the bandpass of the narrowband cometary filters (Farnham et al. 2000). The upper limits to the fluxes are listed in Table 5. The derived upper limit to the gas production rates of the most detected neutrals are also listed in Table 5. Real measurements of fluxes were provided for emission features that were detected in the spectra obtained in December 2008. The related gas production rates are given in Table 5, as well.

\section{Discussion}

Spectra of comets rarely show rich molecular components at large heliocentric distances before perihelion. There is a great deal in common between W3's spectrum and that of comet C/1995 O1 (Hale-Bopp) that was obtained at comparable distances from the Sun. Emissions of $\mathrm{CN}, \mathrm{C}_{3}, \mathrm{C}_{2}, \mathrm{CH}, \mathrm{NH}_{2}, \mathrm{CO}^{+}$, and $\mathrm{CH}^{+}$were detected in both comets, but some of them appeared in the spectrum of C/1995 O1 (Hale-Bopp), when the comet was at greater distances from the Sun. First detection of $\mathrm{CN}$ at a heliocentric distance of 6.8 au was reported by Fitzsimmons \& Cartwright (1996). $\mathrm{C}_{2}, \mathrm{C}_{3}$, and $\mathrm{NH}_{2}$ appeared at a heliocentric distance of 4.6 au (Rauer et al. 1997). However, other molecules in the coma of comet C/1995 O1 (Hale-Bopp) were observed closer to the Sun. The weak emissions from $\mathrm{CH}$ and $\mathrm{CH}^{+}$appeared at 3.1 au (Rauer et al. 1997). The presence of $\mathrm{CO}^{+}$at a heliocentric distance of 3.4 au was first reported by Jockers et al. (1996) and then confirmed by Rauer et al. (1997). Unfortunately, Rauer et al. (1997) did not provide any quantitative measurements for $\mathrm{CO}^{+}$. Nevertheless, by comparing the peak flux of the $\mathrm{CO}^{+}(2-0)$ band in W3's spectrum and that for C/1995 O1 (Hale-Bopp) (see Fig. 3c in Rauer et al. 1997) we can conclude that $\mathrm{W} 3$ is more $\mathrm{CO}^{+}$rich, and, consequently, its activity is mainly controlled by the sublimation of $\mathrm{CO}$ at this distance. This finding is consistent with measurements provided by Bockelée-Morvan et al. (2010) and Ootsubo et al. (2012).

The calculated production rates are not as small for $\mathrm{W} 3$ as for C/1995 O1 (Hale-Bopp). Rauer et al. (1997) reported gas production rates of $6.0 \times 10^{26} \mathrm{~mol} \mathrm{~s}^{-1}$ for $\mathrm{CN}, 7.0 \times 10^{26} \mathrm{~mol} \mathrm{~s}^{-1}$ for $\mathrm{C}_{2}$, and $4.0 \times 10^{26} \mathrm{~mol} \mathrm{~s}^{-1}$ for $\mathrm{NH}_{2}$, when $\mathrm{C} / 1995 \mathrm{O} 1$ (Hale-Bopp) was at a heliocentric distance of $4.0 \mathrm{au}$. We derived $1.2 \times 10^{26} \mathrm{~mol} \mathrm{~s}^{-1}$ for $\mathrm{CN}, 0.7 \times 10^{26} \mathrm{~mol} \mathrm{~s}^{-1}$ for $\mathrm{C}_{2}$, and $0.3 \times 10^{26} \mathrm{~mol} \mathrm{~s}^{-1}$ for $\mathrm{NH}_{2}$ for $\mathrm{W} 3$ at a heliocentric distance of $3.74 \mathrm{au}$. This is not surprising, since the size of the nucleus of W3 is estimated to be a factor two smaller than the size of C/1995 O1 (Hale-Bopp), i.e. $D \sim 26 \mathrm{~km}$ for $\mathrm{W} 3$ was derived from our data in agreement with Bockelée-Morvan et al. (2010).

At large heliocentric distances, emissions of $\mathrm{CO}^{+}$have so far only been detected in a few comets: C/1961 R1 (Humason) (Dossin 1966), 29P/Schwassmann-Wachmann 1 (Cochran et al. 1980, 1982, 1992; Cochran \& Cochran 1991; Korsun et al. 2008), C/1995 O1 Hale-Bopp (Jockers et al. 1996), and C/2002 VQ94 (LINEAR) (Korsun et al. 2006, 2014). Analysis of $\mathrm{CO}^{+}$ coma and estimation of column density of $\mathrm{CO}^{+}$at large heliocentric distances are rare in the cometary history and performed only for comet 29P/Schwassmann-Wachmann 1 (Jockers et al. 1992) and for comet C/2002 VQ94 (LINEAR) (Ivanova et al. 2009; Korsun et al. 2008), when the comets were at heliocentric distances of 5.8 and 7.3 au, respectively.

Production rates of $\mathrm{NH}_{2}$ are often used to derive $\mathrm{NH}_{3}$ abundance in comets (Tegler \& Wyckoff 1989). Since it is expected that the photolytic reaction

$\mathrm{NH}_{3}+h v \rightarrow \mathrm{NH}_{2}+\mathrm{H}$

has a $95 \%$ branching ratio (Allen et al. 1987) and $\mathrm{NH}_{3}$ is the main parent for $\mathrm{NH}_{2}$, then a production rate derived for $\mathrm{NH}_{2}$ is essentially a direct measurement of the production rate of $\mathrm{NH}_{3}$ in comets. We obtained $Q\left(\mathrm{NH}_{2}\right)=3.12 \times 10^{25} \mathrm{~mol} \mathrm{~s}^{-1}$ for W3 at a heliocentric distance of 3.74 au. No emissions of $\mathrm{NH}_{3}$ were detected with the Herschel Space Observatory when the comet was at a distance of 5.0 au from the Sun and the upper limits of $Q\left(\mathrm{NH}_{3}\right)<1.5-1.9 \times 10^{27} \mathrm{~mol} \mathrm{~s}^{-1}$ were only derived (de Val-Borro et al. 2014). We can at least conclude that our data do not contradict these measurements and provide more accurate value of the gas production rate of ammonia for W3.

Previous measurements revealed that the gas production rate of W3 at large heliocentric distances was controlled by the sublimation of highly volatile CO (Bockelée-Morvan et al. 2010; de Val-Borro et al. 2014). The physical mechanisms causing gas release from a comet nucleus are likely changed with its distance from the Sun. According to Bar-Nun et al. (1987), the annealing 
Table 5. Upper limits and real measurements for the main cometary species in the spectrum of comet W3.

\begin{tabular}{|c|c|c|c|c|c|}
\hline Molecule & $\begin{array}{c}\text { Central wavelength } \\
/ \Delta \lambda(\AA)\end{array}$ & $\begin{array}{c}\text { Date of } \\
\text { observations }\end{array}$ & $\begin{array}{l}\text { Amplitude } \times 10^{-17} \\
\left(\mathrm{erg} \mathrm{s}^{-1} \mathrm{~cm}^{-2} \AA^{-1}\right)\end{array}$ & $\begin{array}{l}\text { Flux } \times 10^{-17} \\
\left(\mathrm{erg} \mathrm{s}^{-1} \mathrm{~cm}^{-2}\right)\end{array}$ & $\begin{array}{l}\text { Gas production } \\
\text { rate }\left(10^{24} \mathrm{~mol} \mathrm{~s}^{-1}\right)\end{array}$ \\
\hline \multirow[t]{3}{*}{$\mathrm{CN}$} & $3870 / 62$ & Dec. 13,2006 & $<0.56$ & $<6.2$ & $<1.45$ \\
\hline & & Nov. 9, 2007 & $<1.13$ & $<15.7$ & $<2.92$ \\
\hline & & Dec. 4, 2008 & & 11733.7 & 102.66 \\
\hline \multirow[t]{3}{*}{$\mathrm{C}_{3}$} & $4062 / 62$ & Dec. 13,2006 & $<0.48$ & $<5.3$ & $<0.248$ \\
\hline & & Nov. 9, 2007 & $<0.72$ & $<7.9$ & $<0.162$ \\
\hline & & Dec. 4, 2008 & & 4221.2 & 4.12 \\
\hline \multirow[t]{3}{*}{$\mathrm{CO}^{+}$} & $4266 / 64$ & Dec. 13,2006 & $<0.41$ & $<4.5$ & \\
\hline & & Nov. 9, 2007 & $<0.73$ & $<8.0$ & \\
\hline & & Dec. 4, 2008 & & 720.7 & \\
\hline \multirow[t]{3}{*}{$\mathrm{C}_{2}$} & $5141 / 118$ & Dec. 13,2006 & $<0.24$ & $<2.6$ & $<1.24$ \\
\hline & & Nov. 9, 2007 & $<0.59$ & $<6.5$ & $<1.71$ \\
\hline & & Dec. 4, 2008 & & 5771.2 & 65.42 \\
\hline \multirow[t]{3}{*}{$\mathrm{NH}_{2}$} & $5715 / 90$ & Dec. 13,2006 & $<0.19$ & $<2.1$ & $<6.73$ \\
\hline & & Nov. 9, 2007 & $<0.39$ & $<4.3$ & $<6.01$ \\
\hline & & Dec. 4, 2008 & & 472.1 & 3117 \\
\hline
\end{tabular}

of the amorphous ice can be efficient in a temperature range between 80 and $130 \mathrm{~K}$. The transformation of the ice matrix from amorphous ice to cubic ice can create a vigorous gas release and sustain physical activity in a temperature range of between 130 and $150 \mathrm{~K}$ (Bar-Nun et al. 1987). According to our measurements, a steep growth of activity of W3 occurred around $3.96 \mathrm{au}$, which roughly corresponds to a temperature of $150 \mathrm{~K}$. The evolution of the W3 optical spectra shows the presence of molecular emissions at 3.74 au. Ootsubo et al. (2012) report the measurements of the $\mathrm{H}_{2} \mathrm{O}, \mathrm{CO}_{2}$, and $\mathrm{CO}$ gas production rates with the AKARI satellite (centered at 2.7, 4.3, and $4.7 \mu \mathrm{m}$ ) in December 2008 and June 2009. Between these two observation periods, which roughly coincided with our observing runs in 2008 and 2009, the measured production rate of $\mathrm{H}_{2} \mathrm{O}$ increased from $83.0 \pm 8.5$ to $(201.1 \pm 20.3) \times 10^{26} \mathrm{~mol} \mathrm{~s}^{-1}$, while the $\mathrm{CO}_{2}$ production rate stayed at the same level and the $\mathrm{CO}$ production rate decreased.

The calculated pre-perihelion dust production rate at $3.13 \mathrm{au,}$ $380 \mathrm{~kg}^{-1}$, is about three times lower than the post-perihelion one inferred from the PACS (Photodetector Array Camera and Spectrometer) 70 and $160 \mu \mathrm{m}$ images onboard the Herschel Space Observatory at 3.34 au (de Val-Borro et al. 2014). Dust production rates of $923 / 676 \mathrm{~kg} \mathrm{~s}^{-1}$ and $884 / 965 \mathrm{~kg} \mathrm{~s}^{-1}$ were derived for carbon/olivine grains in 70 and $160 \mu \mathrm{m}$, respectively. The postperihelion PACS measurements of the dust production at 5 au $\left(70-110 \mathrm{~kg} \mathrm{~s}^{-1}\right)$ is closer to the pre-perihelion dust production rate obtained in this study $\left(20-100 \mathrm{~kg} \mathrm{~s}^{-1}\right)$ for heliocentric distances between 6.24 and 3.96 au before the perihelion passage (de Val-Borro et al. 2014).

\section{Conclusions}

We present results from the spectrophotometric monitoring of the long period comet C/2006 W3 (Christensen). The observations were conducted from December 2006 to June 2009 when the comet moved between 8.49 and 3.13 au before the perihelion passage. Our optical observations complements the data set obtained with the Spitzer, Herschel Space Observatory in infrared and far infrared, as well as with ground-based radio-telescopes in the range of heliocentric distances from 3.13 au before perihelion passage to $5.0 \mathrm{au}$ after perihelion passage. Thus the overall orbital arc covered by observations contains the interval from 8.4 au before perihelion to 5.0 au after perihelion. The main results provided by our study can be summarized as follows:

1. No emission lines were detected in the comet spectrum at the distances 8.49 and 6.25 au from the Sun; meanwhile an emission rich spectrum was obtained when the comet moved at a heliocentric distance of 3.74 au. Emissions of $\mathrm{CN}, \mathrm{C}_{3}$, $\mathrm{C}_{2}, \mathrm{CH}, \mathrm{NH}_{2}, \mathrm{CO}^{+}$, and $\mathrm{CH}^{+}$were detected in the spectrum.

2. The $\mathrm{CO}^{+}$emissions, which were registered at a distance of 3.74 au from the Sun, provide evidence that the comet is $\mathrm{CO}$ and/or $\mathrm{CO}_{2}$ enriched. At large heliocentric distances, $\mathrm{CO}^{+}$ ions have only been detected in a few comets so far, e.g. C/1961 R1 (Humason), 29P/Schwassmann-Wachmann 1, C/1995 O1 Hale-Bopp, and C/2002 VQ94 (LINEAR).

3. Upper limits on gas production rates and gas production rates for the detected neutrals were derived from the spectra with a Haser model.

4. The $\mathrm{CO}^{+}$production rate of the comet equal to $2 \times$ $10^{24} \mathrm{~mol} \mathrm{~s}^{-1}$ was obtained from a map with the column densities of $\mathrm{CO}^{+}$.

5. The photometric results indicate that $\mathrm{W} 3$ was active from our earliest observations at 8.49 au. Dependence of $A f \rho$ on heliocentric distance reveals steep growth of the W3 activity around 3.96 au before perihelion passage.

6. The normalized reflectivity gradient of the inner coma varies between $2 \% / 1000 \AA$ and $5 \% / 1000 \AA$, increasing to $10 \% / 1000 \AA$ in the outer part of the coma. The color of the inner part of the $\mathrm{W} 3$ coma is in agreement with measurements reported for the sample of long period comets.

7. The upper limit on the nucleus radius extracted from the photometric measurements, $R_{N}=13 \pm 2 \mathrm{~km}$, is in agreement with previous results obtained from the Herschel infrared data set.

Acknowledgements. The results are based on the observations made with the $6 \mathrm{~m}$ telescope of SAO RAN and the $2 \mathrm{~m}$ telescope of Peak Terskol Observatory. The observations at the $6 \mathrm{~m}$ BTA telescope were carried out with the financial support of the Ministry of Education and Science of the Russian Federation (agreement No. 14.619.21.0004, project ID RFMEFI61914X0004). This work was also supported by the Ministry of Education and Science of Ukraine (agreement No. GP/F56/ 078). The authors thank the anonymous reviewer for valuable comments on the manuscript. 


\section{References}

Afanasiev, V. L., \& Moiseev, A. V. 2005, Astron. Lett., 31, 194 Ahearn, M. F., Schleicher, D. G., Millis, R. L., Feldman, P. D., \& Thompson, D. T. 1984, AJ, 89, 579

A’Hearn, M. F., Millis, R. C., Schleicher, D. O., Osip, D. J., \& Birch, P. V. 1995, Icarus, 118, 223

Allen, M., Delitsky, M., Huntress, W., Yung, Y., \& Ip, W.-H. 1987, A\&A, 187, 502

Bar-Nun, A., Dror, J., Kochavi, E., \& Laufer, D. 1987, Phys. Rev. B, 35, 2427

Bessell, M. S. 1999, PASP, 111, 1426

Bockelée-Morvan, D., Hartogh, P., Crovisier, J., et al. 2010, A\&A, 518, L149

Bonev, T., \& Jockers, K. 2002, in Asteroids, Comets, and Meteors: ACM 2002, ed. B. Warmbein, ESA SP, 500, 587

Bonev, T., Jockers, K., Petrova, E., et al. 2002, Icarus, 160, 419

Buzzi, L., Luppi, F., Stevens, B. L., et al. 2006, Minor Planet Electronic Circulars

Cochran, A. L., \& Cochran, W. D. 1991, Icarus, 90, 172

Cochran, A., Barker, E. S., \& Cochran, W. 1980, AJ, 85, 474

Cochran, A. L., Cochran, W. D., \& Barker, E. S. 1982, ApJ, 254, 816

Cochran, A. L., Barker, E. S., Ramseyer, T. F., \& Storrs, A. D. 1992, Icarus, 98 , 151

de Val-Borro, M., Bockelée-Morvan, D., Jehin, E., et al. 2014, A\&A, 564, A124

Dossin, F. V. 1966, AJ, 71, 853

Farnham, T. L., Schleicher, D. G., \& A'Hearn, M. F. 2000, Icarus, 147, 180

Fitzsimmons, A., \& Cartwright, I. M. 1996, MNRAS, 278, L37

Fukugita, M., Ichikawa, T., Gunn, J. E., et al. 1996, AJ, 111, 1748

Haser, L. 1957, Bull. Soc. R. Sciences de Liège, 43, 740

Ivanova, A. V., Korsun, P. P., \& Afanasiev, V. L. 2009, Solar System Research, 43,453

Jewitt, D. 2015, AJ, 150, 201

Jewitt, D., \& Meech, K. J. 1986, ApJ, 310, 937

Jockers, K., Bonev, T., Ivanova, V., \& Rauer, H. 1992, A\&A, 260, 455

Jockers, K., Credner, T., Karpov, N., \& Sergeev, A. 1996, IAU Circ., 6468

Kartasheva, T. A., \& Chunakova, N. M. 1978, Astrofizicheskie Issledovaniia Izvestiya Spetsial'noj Astrofizicheskoj Observatorii, 10, 44

Korsun, P. P., Ivanova, O. V., \& Afanasiev, V. L. 2006, A\&A, 459, 977

Korsun, P. P., Ivanova, O. V., \& Afanasiev, V. L. 2008, Icarus, 198, 465
Korsun, P. P., Kulyk, I. V., Ivanova, O. V., et al. 2010, Icarus, 210, 916

Korsun, P. P., Rousselot, P., Kulyk, I. V., Afanasiev, V. L., \& Ivanova, O. V. 2014, Icarus, 232, 88

Kossacki, K. J., \& Szutowicz, S. 2015, Icarus, 250, 595

Krishna Swamy, K. S. 2010, Physics of comets (Singapore; Hackensack, N. J: World Scientific Pub. Co.)

Kulyk, I., Jockers, K., Credner, T., \& Bonev, T. 2004, Kinematika i Fizika Nebesnykh Tel, 20, 372

Kulyk, I., Korsun, P., Rousselot, P., Afanasiev, V., \& Ivanova, O. 2016, Icarus, 271, 314

Lamy, P. L., Toth, I., Fernandez, Y. R., \& Weaver, H. A. 2004, The sizes, shapes, albedos, and colors of cometary nuclei, eds. M. C. Festou, H. U. Keller, \& H. A. Weaver, 223

Landolt, A. U. 1992, AJ, 104, 340

Lutz, B. L., Womack, M., \& Wagner, R. M. 1993, ApJ, 407, 402

McKay, A. J., Chanover, N. J., Morgenthaler, J. P., et al. 2012, Icarus, 220, 277

Merline, W. J., \& Howell, S. B. 1995, Exp. Astron., 6, 163

Neckel, H., \& Labs, D. 1984, Sol. Phys., 90, 205

Newburn, R. L., \& Spinrad, H. 1985, AJ, 90, 2591

Oke, J. B. 1990, AJ, 99, 1621

Ootsubo, T., Kawakita, H., Hamada, S., et al. 2012, ApJ, 752, 15

Rauer, H., Arpigny, C., Boehnhardt, H., et al. 1997, Science, 275, 1909

Reach, W. T., Kelley, M. S., \& Vaubaillon, J. 2013, Icarus, 226, 777

Rousselot, P., Korsun, P. P., Kulyk, I. V., et al. 2014, A\&A, 571, A73

Russell, H. N. 1916, ApJ, 43, 173

Schleicher, D. G. 2010, AJ, 140, 973

Schleicher, D. G., \& Farnham, T. L. 2004, Photometry and imaging of the coma with narrowband filters, ed. G. W. Kronk, 449

Sekanina, Z., Larson, S. M., Hainaut, O., Smette, A., \& West, R. M. 1992, A\&A, 263, 367

Singh, P. D., de Almeida, A. A., \& Huebner, W. F. 1992, AJ, 104, 848

Stetson, P. B. 1987, PASP, 99, 191

Tancredi, G., Fernández, J. A., Rickman, H., \& Licandro, J. 2000, A\&AS, 146, 73

Tegler, S., \& Wyckoff, S. 1989, ApJ, 343, 445

Weiler, M., Rauer, H., Knollenberg, J., Jorda, L., \& Helbert, J. 2003, A\&A, 403, 313 